\title{
Safety and feasibility of myomectomy during caesarean section in case of pregnancy associated with fibroid uterus
}

\author{
SF Yeasmin ${ }^{1 凶}$, MA Hasanat ${ }^{2}$, E Saha $^{3}$, MT Rahman $^{4}$, DK Sunyal ${ }^{5}$
}

\begin{abstract}
Routine myomectomy during pregnancy is not recommended because of excessive bleeding during myomectomy and the difficulty in securing hemostasis may need hysterectomy. This prospective observational study was carried out in Obstetrics \& Gynecology department of Ad-din Akij Medical College, Khulna from June 2016 to August 2017 to evaluate safety and feasibility of performing myomectomy at the time of caesarean delivery. Nineteen patients with fibroid uterus with pregnancy whom needed cesarean section for various indications were the target population for this study. Main outcome measures were difficulty of myomectomy \& cesarean section, time needed for operation, per-operative complications, need for blood transfusion, postoperative complications and duration of hospital stay. Caesarean myomectomy operation was successful in all cases. No patient was required hysterectomy. The duration of operation was 45-60 minutes. Only one patient had one unit of whole blood transfusion during postoperative period. Mean (SD) duration of hospital stay and post-operative $\mathrm{Hb}$ concentration were $6.9(0.6)$ days and $8.6(1.4) \mathrm{g} / \mathrm{dl}$, respectively. Two patients developed post-operative fever which delayed their hospital stay. Myomectomy during cesarean section in selected cases with proper indication is safe and feasible.
\end{abstract}

Key words: caesarean section, myomectomy, safety, feasibility, hemostasis.

\section{Introduction}

Fibroids are benign tumors of the uterine smooth muscle. They commonly occur in women of childbearing age. According to various studies the incidence of uterine fibroids during pregnancy ranges from 1.6 to $10.7 \%$ cases. Usually leiomyomas are asymptomatic but their maximum growth rate is in late reproductive years and affects pregnancy and child birth. Pregnancy associated with uterine leiomyoma increases the rate of cesarean section six fold higher while compared with normal unaffected gestation. ${ }^{1}$ But routine myomectomy during pregnancy is not recommended because of size and increased vascularity of myoma and there may be excessive bleeding during myomectomy, and the difficulty in securing homeostasis may need hysterectomy. ${ }^{2}$

The rate of cesarean sections, age at pregnancy and gravida have been gradually increasing, and thus, the chances of an obstetrician to encounter uterine fibroids

\footnotetext{
1. SF Yeasmin, MBBS, DGo, Assistant Professor of Obstetrics \& Gynaecology, Ad-din Akij Medical College, Khulna. Email: farzana.yesmin@gmail.com

2. MA Hasanat, MBBS, M Phil, Assistant Professor of Physiology, Ad-din Akij Medical College, Khulna

3. E Saha, MBBS, FCPS, Associate Professor of Obstetrics \& Gynaecology, Khulna Medical College, Khulna

4. MT Rahman, MBBS, MD, Consultant (Internal Medicine), Fulbaria Upazila Health Complex, Mymensingh

5. DK Sunyal, MBBS, MPhil, Professor of Physiology, Gazi Medical College, Khulna
} 
among their patients during cesarean section have increased. ${ }^{3}$ Newer studies describe the advantages of myomectomy during cesarean section. In the postpartum phase, uterus is better adapted physiologically to control hemorrhage as contractions and retractions of muscle fibers occur making the blood vessels closed. Also the onset of vascular changes for clot formation in placental bed helps in stopping the bleeding. ${ }^{4}$ Moreover, enucleation of myoma during cesarean myomectomy is easier as the capsule becomes loose. It helps to avoid the risk of another surgery and anesthesia for removal of myomas. It also facilitates the chances of vaginal delivery in subsequent pregnancies when myoma removed from lower uterine segment. ${ }^{2}$

Therefore, the present study was designed to evaluate the safety and feasibility of doing myomectomy during cesarean section.

\section{Materials and Method}

This prospective observational study was carried out in the Department of Obstetrics \& Gynaecology of Ad-din Akij Medical College, Khulna from June 2016 to August 2017. Nineteen patients with fibroid uterus with pregnancy whom needed cesarean section for various indications were included as study population. Diagnoses of uterine fibroid were established by ultrasonography during antenatal check-up or incidentally diagnosed to have fibroid during cesarean section. Proper evaluation of location, number, size and position of fibroids was done. Patients were informed about the risks and benefits of myomectomy during cesarean section and informed consents were taken before operation and during operation in incidental cases. Most of the cases had cesarean section at term for various obstetrics indications. Oxytocin infusion was started after delivery of the baby and continued for 24 hours thereafter. Main outcome measures were: difficulty in myomectomy \& cesarean section, time needed for operation, peroperative complications, need for blood transfusion, postoperative complications and duration of hospital stay.
Results

During the study period 19 women underwent cesarean myomectomy for various indications. Table 1 shows that fibroid uterus was more common in pregnancy in ages between $28-35$ years (52.6\%).

The Incidence rate of fibroid in pregnancy is higher among the multiparous and in 16 cases caesarean myomectomy was done at term pregnancy $(84.2 \%)$ and there was no history of abortion in 17 cases (89.5\%) (Table 2).

Table 3 shows the number, type, size and location of the fibroids. Seven cases had only one or two fibroids (36.8\%) and 12 cases had multiple fibroid $(63.2 \%)$. The commonest type of fibroids was subserous (73.7\%). Maximum fibroids were less than $5 \mathrm{~cm}$. In majority of the cases, fibroids were located on the body of the uterus $(78.9 \%)$.

Table 4 shows the intra-operative/ postoperative outcomes in this study. For most of the cases $(94.7 \%)$, the duration of operation was 45-60 minutes. Only one case required blood transfusion. No patient had life-threatening hemorrhage in this study and none had a hysterectomy as a result of excessive blood loss. Only two patients developed post-operative fever which delayed their hospital stay. Maximum cases $(89.5 \%)$ were discharged from hospital like normal post caesarean patients and the mean (SD) post-operative $\mathrm{Hb}$ concentration was $8.6(1.4) \mathrm{g} / \mathrm{dl}$.

\section{Discussion}

Fibroids are the commonest tumors in our female population. Also coexisting of fibroids with complication of pregnancy is not an uncommon presentation to obstetrician practicing in our country. ${ }^{5}$ Many women are delaying child bearing till their late thirties, which is the time for greatest risk of myoma growth. Use of ultrasound has improved the diagnostic capability of detecting small myomas and has increased knowledge of myoma in pregnancy. ${ }^{6}$ The majority of myomas associated with pregnancy remain asymptomatic and do not require treatment, 
Table 1. Distribution of mothers according to age, $n=19$

\begin{tabular}{lrr}
\hline Age (years) & Number & $\%$ \\
\hline $20-27$ & 8 & 42.1 \\
$28-35$ & 10 & 52.6 \\
$>35$ & 1 & 5.3 \\
\hline
\end{tabular}

Table 2. Case distribution according to obstetric history, $n=19$

\begin{tabular}{lrr}
\hline Case distribution & Number & $\%$ \\
\hline Parity & & \\
$\quad$ Primigravida & 6 & 31.6 \\
Para 1 & 9 & 47.4 \\
Para 2 & 3 & 15.8 \\
Para 3 & 1 & 5.3 \\
Gestational ages & & \\
$\quad$ Preterm & 3 & 15.8 \\
$\quad$ Term & 16 & 84.2 \\
History of abortion & & \\
$\quad$ Yes & 2 & 10.5 \\
No & 17 & 89.5 \\
\hline
\end{tabular}

Table 3. Number, type, size and location of the fibroids, $\mathbf{n}=19$

\begin{tabular}{lrr}
\hline Case distribution & Number & $\%$ \\
\hline Number of fibroids & 7 & 36.8 \\
$1-2$ & 12 & 63.2 \\
Multiple & & \\
Type of fibroids & 14 & 73.7 \\
$\quad$ Subserous & 5 & 26.3 \\
$\quad$ Intramural & & \\
Size of fibroids & 17 & 89.5 \\
$\quad<5 \mathrm{~cm}$ & 2 & 10.5 \\
$>5 \mathrm{~cm}$ & & \\
Location of fibroids & 15 & 78.9 \\
$\quad$ Body of uterus & 4 & 21.1 \\
$\quad$ Fundus & & \\
\hline
\end{tabular}

Table 4. Intra-operative/ post-operative outcomes in this study, n = 19

\begin{tabular}{lrr}
\hline Outcome & Number & \% or Mean (SD) \\
\hline Duration of surgery, minutes & & \\
$\quad 45-60$ & 18 & 94.7 \\
$\quad>60$ & 1 & 5.3 \\
Needed blood transfusion & & \\
$\quad$ Yes & 1 & 5.3 \\
$\quad$ No & 18 & 94.7 \\
Duration of hospital stay, days & & $6.9(0.5)$ \\
$\quad 0-7$ & 17 & 89.5 \\
$>7$ & 2 & 10.5 \\
Post operative Hb concentration, g/dl & & $8.6(1.4)$ \\
\hline
\end{tabular}


with about $22-32 \%$ showing increased growth. Larger fibroids $(>5 \mathrm{~cm})$ are more likely to grow during pregnancy and can cause miscarriages, obstructed labour, malpresentations, pressure symptoms, pain due to red degeneration, preterm labour, preterm premature rupture of membranes, retained placenta, postpartum haemorrhage and uterine torsion. Preservation of the uterus without loss of its function and compromising the mother's ability to bear more children is definitely a greater surgical achievement than a hysterectomy; hence, caesarean myomectomy must be considered by experienced obstetricians wherever feasible. ${ }^{7}$

Myomectomy has traditionally been discouraged during cesarean delivery due to high risk of hemorrhage associated with this procedure and need for blood transfusion. On the other hand, if myomectomy is avoided, it may increase the patient's risk of another operation that may unfavorably affect future pregnancies. If safely performed, the procedure is cost-effective and can prevent morbidities associated with potential future surgeries. Myomectomy also reduces puerperal uterine involution and could decrease complications related to uterine fibroids, such as menorrhagia, anemia, and pain. Several recent studies have described techniques which can minimize blood loss at cesarean myomectomy, including uterine tourniquet, bilateral uterine artery ligation, and electro-cautery, although none of these techniques were used in our study, they may further reduce blood loss during cesarean myomectomy. ${ }^{3,8} \mathrm{~A}$ study reported 18 cases of myomectomy during pregnancy 16 of them delivered uneventfully at term. ${ }^{9}$ Other studies have reported similar results with a good perinatal outcome but also reported an increased preterm delivery rate. ${ }^{10,11} \mathrm{~A}$ comparative study compared the outcomes of 111 patients who had myomectomy at caesarean with 257 patients who had undergone caesarean alone. No significant difference was found in the incidence of intra or postoperative complications between the two groups. ${ }^{8}$
Some previous studies have reported that it is better to avoid myomectomy during cesarean section except for small pedunculated fibroid.2 In a study conducted on 14 cases in India, the average duration of operation was longer in cases having myomectomy with LSCS (54.1 minutes) than in those who had LCSC only (35 minutes).12 But the fact and newer studies described the advantages of myomectomy during cesarean section. These are myomectomy during caesarean section would cause less bleeding because at post-partum the uterus is better adopted for control of hemorrhage as the uterine muscles are more sensitive to oxytocic agents and removal of myoma enable the uterus for better contraction. Moreover, the pregnant patients are in hypervolumic and hypercoagulable state, so can cope easily with a certain amount of blood loss.2 Our study also shows that cesarean myomectomy is not as dangerous as generations of obstetricians have been trained to believe. Enucleation of the fibroid is technically easier in gravid uterus owing to greater looseness of the capsule. Retraction of uterine muscles is enhanced by oxytocic agents to help arrest the hemorrhage.

\section{Conclusion}

Overall cesarean myomectomy may be a reasonable and safety option by experienced obstetrician. Due to improvement of surgical skill, surgical technique, availability of blood and anesthetic procedure, it has become possible to manage the cases with severe intra-partum hemorrhage. Proper de-vascularization and availability of an experienced obstetrician can reduce the incidence of hemorrhage and hysterectomy.

\section{References}

1. Song D. Myomectomy during cesarean section is likely feasible. Clinics Mother Child Health 2016;13(2):1-3.

2. Baby HA, Begum MR, Ehsan $M$, et al. Myomectomy during caesarean section: safety and feasibility of the procedure. Bangladesh J Obstet Gynaecol 2015;30(1):10-4.

3. Senturk MB, Budak MS, Cakmak Y, Turan 
$\mathrm{K}$. Is myomectomy safe during cesarean section in large myomas? Medical Science and Discovery 2015;2(5):292-6.

4. Anita K, Seema M, Richa R. Cesarean myomectomy. J Obstet Gynecol India 2007;57(2):128-30.

5. Awoleke JO. Myomectomy during caesarean birth in fibroid-endemic, low resource settings. Obstet Gynecol Int 2013:520834.

6. Biswas SP, Fatema MK, Akhter S. Safety of routine caesarean myomectomy. Bang Med J Khulna 2013;46:7-11.

7. Machado LS, Gowri V, Al-Riyami N, Al-Kharusi L. Caesarean myomectomy: feasibility and safety. Sultan Quaboos Univ Med J 2012;12(2):190-6.

8. Roman AS, Tabsh KMA. Myomectomy at time of caesarean delivery; a retrospective cohort study. BMC Pregnancy Child Birth 2004;4:14-7.

9. Michalas SP, Oreopoulou FV, Papageorgiou JS. Myomectomy during pregnancy and cesarean section. Hum Reprod 1995;10:1869-70.

10. Burton CA, Grimes DA, March CM. Surgical management of leiomyoma during pregnancy. Obstet Gynecol 1989;74:707-9.

11. Exacoustos C, Rosati P. Ultrasound diagnosis of uterine myomas and complications in pregnancy. Obstet Gynecol 1993;82:97-101.

12.Sudhir A, Sebanti G. Cesarean myomectomy- a study of 14 cases. J Obstet Gynecol India 2006;56(6):486-8.

\section{Suggestion for citation of the above:}

Yeasmin SF, Hasanat MA, Saha E, Rahman MT, Sunyal DK. Safety and feasibility of myomectomy during caesarean section in case of pregnancy associated with fibroid uterus. Mediscope 2018;5(1):5-9. 